\title{
Getting to grips with Supplementary Information
}

\section{Nature Cell Biology implements new guidelines for Supplementary Information.}

The growth and unstructured nature of Supplementary Information has become a serious concern for cell biologists. The proliferation of information in the supplemental section has made it cumbersome for readers to access and navigate. All too often, it can become a dump for premature experiments addressing issues that are not directly pertinent to the central focus of the study, which can contribute to an unevenness in data quality when compared with the main text. Conversely, in short-format research papers with a more restricted number of main-text figures, key data that ought to be in the main figures can get buried in the supplemental section. Another concern is that the combined volume of data in the main text and the Supplementary Information is sometimes equivalent to that in multiple research papers, thus greatly increasing the burden on referees. Authors, referees and readers have consistently raised these concerns in the past few years.

Some journals have responded by abolishing Supplementary Information altogether. However, we believe that the supplemental section can enrich a published paper, and so removing it entirely is not an option for this journal. Our research papers have become increasingly multifaceted; they often address topics at the juncture of several areas in cell biology, employ a diverse range of approaches, including high-throughput assays with their associated large data sets, and often combine cell-culture data with in vivo whole-organism experiments. As the complexity and variety of the data in the main text has increased, there is a greater need for a supplemental section that functions as an addendum, providing additional supporting data that bolster the central claims of the study, additional validation of the methodology, or additional data that is likely to be largely of specialist interest but nevertheless enriches the study. For the general reader, the main text should suffice as a clearly articulated research paper, whereas the Supplementary Information can provide a secondary resource for the specialist reader who may want to delve deeper into the specific findings. Material that is appropriate for the supplement includes data that characterizes reagents, replicate experiments, additional control data, and lists of primers, RNAi sequences and plasmids used in the study. Standalone methodology-related descriptions, for example of software algorithms or reagent characterization, may also be provided as Supplementary Information.

In light of these considerations, we have introduced new guidelines for Supplementary Information that limits the total number of figures. These guidelines stipulate that the number of Supplementary Information figures must not exceed the total number of figures allowed in the main text. Article, Letter and Brief Communication formats allow up to eight, five and two Supplementary Information figures, respectively. Resource papers with large data sets often require additional validation of reagents or extensive display of representative images, and so we will not be limiting the number of supplementary figures for this type of paper. Although limits are currently not imposed on supplementary tables and movies, authors are strongly encouraged to be selective when including these data and to ensure that they are directly related to the study. The data in Supplementary Information must be discussed in the text of the main article, and where possible, each supplementary figure, table or movie must be linked directly to individual main-text figures to form a cohesive narrative. Supplementary text, including additional results or discussions, is not permitted. These changes are now outlined in our Guide to Authors.

We are aware of authors' concerns that growing Supplementary Information sections can also encourage requests for a seemingly endless list of new experiments, some of which might unproductively extend the study beyond the core concept and result in multiple revisions. We will also inform referees of these updated guidelines, and, as always, Nature Cell Biology editors will continue to work with authors and referees to determine the scope of necessary revisions.

Another common complaint from readers is that Supplementary Information is scattered and difficult to find. For several years, this journal has been integrating Supplementary Information figures and other printable data types (excluding spreadsheets and large tables) into the PDF of the manuscript, and enabling oneclick access to a large portion of the Supplementary Information associated with a published paper. This feature has elicited unanimously positive feedback from authors and readers, and so we will continue to provide it. Since November 2011, integration of the Readcube Web Reader on all Nature research journals provides readers with an interactive PDF through a browser-based web reader tool (available under PDF options in the article toolbox). Supplementary Information and associated pieces (such as News \& Views) are also integrated in the PDF viewer. Seamless integration of all Supplementary Information, including video embedding, in HTML versions of published papers is an important future goal to enrich the online reader experience.

We hope that these new guidelines address the most pressing concerns of our readers, authors and referees regarding Supplementary Information. As always, we welcome feedback on changes to editorial policy at cellbio@nature.com. 